Review

\title{
A Strategic Study on the Environmental Impacts of Wind Turbine Blade Materials, their Improvements, Economics and End-Life-Options
}

\author{
${ }^{1}$ Ojshwi Prakash, ${ }^{2}$ Godwin Glivin, ${ }^{2}$ N. Kalaiselvan and ${ }^{3}$ V. Mariappan \\ ${ }^{I}$ Department of Chemical Engineering, National Institute of Technology Tiruchirappalli, Tamil Nadu, India \\ ${ }^{2}$ Department of Energy and Environment, National Institute of Technology Tiruchirappalli, Tamil Nadu, India \\ ${ }^{3}$ Department of Mechanical Engineering, National Institute of Technology Tiruchirappalli, Tamil Nadu, India
}

\author{
Article history \\ Received: 24-08-2020 \\ Revised: 06-11-2020 \\ Accepted: 12-11-2020 \\ Corresponding Author: \\ Ojshwi Prakash \\ Department of Chemical \\ Engineering, National Institute \\ of Technology Tiruchirappalli, \\ Tamil Nadu, India \\ Email: ojshwiprakash@gmail.com
}

\begin{abstract}
Wind energy is a great alternative to solar energy (During monsoon) along with conventional non-renewable energy sources and has a potential market growing exponentially. Modern 3 Blade HAWTs are most dominant in the market and have high efficiency. Blades are 15 to $20 \%$ of the cost of the wind turbine unit and also exposed to the environment. Therefore it is crucial to employ suitable material that has lightweight, high stiffness and strength. Carbon fibre, Glass Fibre, Timber are some materials that are used for blade manufacturing. Design can be improved by stress deformation analysis, load estimation via methodology of blade element momentum or computational simulation using deep learning. Also, by developing a bio-inspirational method via 1\% Gravo-Aeroelastic Scaling (GAS), cavity optimization approach and vacuum infusion or reinforcing resins in composites, to improve mechanical properties. To prevent surface erosion due to acidic or saline or rainwater, it is coated with a protective layer. Mechanical recycling of the blade material composites is both simpler and economically viable in comparison to thermal and chemical recycling.
\end{abstract}

Keywords: Blade Materials, 1\% GAS, Surface Erosion, Economics, Wake Effect, End-Life-Options

\section{Introduction}

The demand for electrical energy is surging exponentially due to rapid population growth and human advances in science and technology. Along with that, the desirable source is the one which has the caliber to deliver emission-free energy and has enormous availability of fuel. Though hydroelectric, nuclear and solar are being used still they have their own environmental and practical drawbacks. Like nuclear power-plants emits harmful gamma radiation, solar parks occupy huge surface lock the usability at a time, that could have been used for agriculture, hydroelectric projects that emit methane due to the submerge of large landmass, risk of tectonic movements, reduce fertility of top soil as river is blocked to carry along the minerals, etc. Though they are eco-friendly, they have specific local requirements and hence can be established only at certain locations. Wind energy is a form of solar energy as wind movements are governed by the pressure or temperature difference created between poles and equator by the solar radiation received by earth. Many onshore and offshore areas possess surplus and regular wind flow. In such places, we can establish wind energy projects that can produce electricity without disturbing the local civilization and allows us to use the land the way we decide. Developing countries like India, which has swiftly growing energy demands can exploit the sites and prevent several thousands of tons of $\mathrm{CO}_{2}$ emission every year. National Institute for Wind Energy estimated the wind energy capacity at height of $100 \mathrm{~m}$ to be about $302 \mathrm{GW}$. At present India is the 4th largest market with current installation generating about $37 \mathrm{GW}$ of electricity by 2019 that accounts to $4 \%$ of net electricity generation. Wind energy though is most effective for 5 months a year which is complementary to solar power. Offshore region has $70 \mathrm{GW}$ capacities along the coasts of Tamil Nadu and Gujrat, though onshore wind energy projects produce relatively cheaper energy. As shown in Fig. 1, there are two types of Wind Turbines (WT) in general:

- Horizontal Axis Wind Turbine (HAWTs)

- Vertical Axis Wind Turbine (VAWTs) 


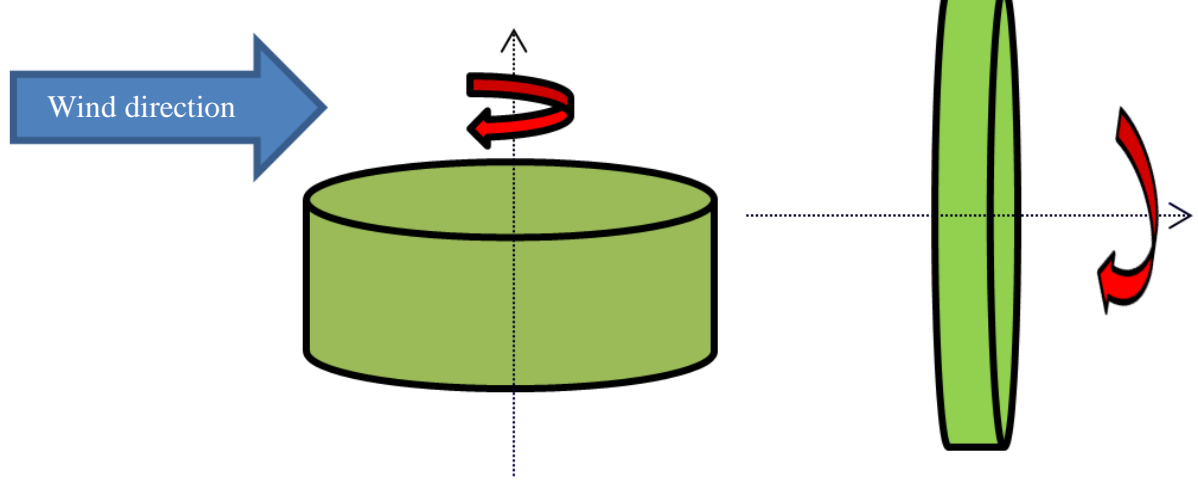

Fig. 1: Horizontal and Vertical configurations for shaft and Rotor

Table 1: Propulsion and Efficiency of Wind Turbine

\begin{tabular}{|c|c|c|c|c|c|c|}
\hline Design & Savonius rotor & Darrieus rotor & Modern wind & & & \\
\hline Orientation & VAWT & VAWT & HAWT & & & \\
\hline \multirow[t]{2}{*}{ Peak efficiency $(\%)$} & 8 & 40 & No. of blades & 1 & 2 & 3 \\
\hline & & & $\mathrm{PF}(\%)$ & 43 & 47 & 50 \\
\hline Propulsion & Drag & Lift & Lift & & & \\
\hline
\end{tabular}

Out of which HAWT prevails being dominant in market and even there 3 blade WTs are used almost everywhere. Europe, essentially Denmark, is reported to be the market leader in WT production and supply. In this study, after a brief discussion on WT, the rotor blade and its material have been discussed. The efficiency of material and after life treatment as well as environmental impact serves to be the main discussion. Wind turbine is has also seen surge in demands after solar and is expected to double in installation capacity by the year 2023. The characteristics of HAWTs and VAWTs have been addressed briefly in Table 1, including their various types.

Hence, their materials must be recycled or shall be chosen with consideration of least negative environmental impact with maximum efficiency (Schubel and Crossley, 2012). The paper includes discussion on the topic wind aerodynamics (wake effect) and wind energy economics. The emphasis is laid over to the blade material, optimal design of the wind turbine blade, environmental impacts upon it and end-life-options.

\section{Wake Effect or Aerodynamics of Wind Turbines}

The study of physical laws, forces and behavior that governs objects in airflow is termed aerodynamics. Required is a design theory that is comprehensive and able to estimate aerodynamic loads that are unsteady and is a result of wind shear via momentum theory. The action of lift and drag over the turbine blades has been shown in Fig. 2, by considering over the cross-section of the WTBs. HAWTs are very dynamic and are subject to aerodynamic loading with complex distributions.
Vertical wind shear can cause motion not only within the vertical plane but also along with the rotor's yaw motion (Herbert et al., 2007).

\section{Wake Effect}

To determine how the lift of an aerofoil actually developed, it's essential to review the wake effect. The various wake effects like:

1. Effect of dynamic loading during the operation of wind turbines within the wake of other results in a rise of extreme load by $50 \%$ and fatigue damage rate of $17 \%$

2. Dynamic inflow models of Coleman and Yaw Dyn accurately predict both the magnitude and thrust center over the rotor disk

To overcome and measure wakes structures, we can use SODAR, computational methods, or a numerical model to calculate the flow field within the wake of the turbine. The experiments in the near wake are going to be assessed by several criteria to judge the significance: A model to tunnel area ratio, Reynolds number, completeness of acquired data. The properties to gauge concern the feasibility to make a comparison between the experimental data and results from computational codes, that the fluid dynamics must be representable for validation. The acceptable rotor properties thus are the aerofoil, the Reynolds number and the construction environment (specifically the tunnel to model area ratio). Far wake models are selected which rely upon the smallest amount simplifying assumptions and hence, are better suited in handling different configurations and in reproducing wake development in additional detail (Vermeer et al., 2003). 


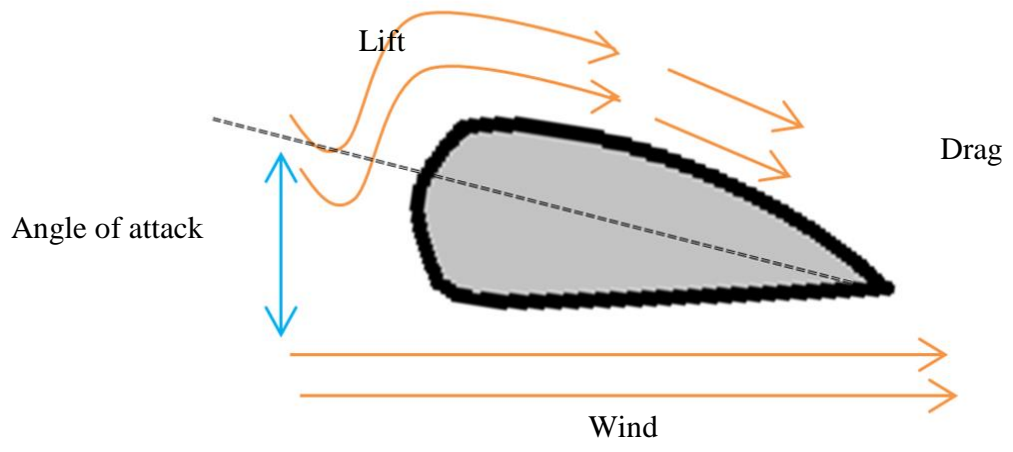

Fig. 2: Wind turbine aerodynamics, lift and drag

\section{Wind Turbine Blade Materials}

\section{Carbon Fibre}

They have high stiffness, anisotropy and properties for thermal expansion, exhibiting more mechanical properties. It is very fine and simply breaks when is stretched or turns into fine dust in the process of handling. These processes are composed of manufacturing to disposal steps. Generally, carbon fiber doesn't undergo combustion; however, the ignition of resin may result in broad distribution of the fibre (Dathu and Hariharan, 2020; Psomopoulos et al., 2019). The emphasis is laid on the economic viability of carbon fiber composites suited for WTB. Carbon fiber is used as it reduces the weight of the blades because of the better strength, fatigue and stiffness, thereby having an advantage over the glass fiber but it relatively more expensive than the glass fiber. They produced in the textile industry and examined under lower blade mass and better fatigue life. Carbon fiber makes it possible to produce more energy at reduced costs (Ennis et al., 2019).

\section{Glass Fibre}

It is mixture of $\mathrm{SiO}_{2}$ and $\mathrm{Al}_{2} \mathrm{O}_{3}$ with addition of different oxides that contributes towards the strength of the fibre. Unlike carbon fibre it is moderately stiff and dense and can sustain high strength (Dathu and Hariharan, 2020). Glass Fibers are more economical in comparison with Carbon fibers.

\section{Timber}

Though composite materials prevail with advantages, timber is widely used in the energy industry, essentially in blades of small turbines. Alder, ash, beech and hornbeam are 4 types of timber. Structural aspects indicate simpler manufacturing of solid blades, but its higher inertia consumes time in the process since small turbines lack pitch mechanisms for optimization of blade angles. The results prove the higher optimal coefficient ability of blades; even then starting performance demands an appropriate choice of timber material. Hence, Alder solid blade is preferred over others whereas alder and beech timbers for the hollow blades for windy regions. All timbers types are promising for areas with lower wind speed and also where starting performance are emphasized (Pourrajabian et al., 2019).

\section{Improving Mechanical Properties}

We can improve the mechanical properties of the fibers by reinforcing composites with nanomaterial like the Carbon Nanotube fibers (CNT), a carbon allotrope is a nanostructure with an excellent aspect ratio greater than $1,000,000$ and their cylindrical orientation are best suited for the wind turbines. The fabrication of CNT is achieved involving different reinforcements to improve properties (Pradeep et al., 2019). Discussions of various features like power produced shear stress with respect to wind speed and characteristics of stress yield versus temperature assists and determine the blade material to be used for optimized energy generation (Murray et al., 2019).

\section{Efficiency}

The best-suited material is required to exhibit features and characteristics like good fatigue strength to provide resistance against the cyclic load, light in weight and high strength to reduce the gravitational and wind forces, high stiffness, etc. Using lightweight materials in WTB, the noise is reduced and the rotation speed is increased though these materials are expensive and generate heat a lot. While medium weight materials reduce noise to some extent, they are long-lasting and their rotation velocity can be more stabilized with respect to the wind speed. Less heat is produced due to smooth rotation. Blade materials such as Copper, Aluminum, Zinc, etc. are long-lasting and rotate smoothly with respect to the wind velocity (Dathu and Hariharan, 2020). Strategic study of power output, in terms of the spatial arrangement of a utility-scale WT array, with Large-Eddy Simulation in the neutrally-stratified atmospheric boundary layer on large WTB, has interesting results. Configurations like perfectly-aligned, laterally-staggered 
and vertically- staggered alignments are considered. Observations shows that staggered alignments tend to generate more power in comparison with aligned configurations. Whereas, laterally staggered configurations produces net more energy, due to better adaptability of the spatial configuration of WTB. However, velocity deficit maxima for vertically staggered configurations, reduces gross turbulence intensity for the wake flow in the wind projects (Wu et al., 2019).

\section{Other Materials for Improved Performance}

The blade materials used in present are generally polyester, glass fiber and carbon fiber, with the addition of other oxides and epoxy material reinforced over the blade. Glass and Carbon Nano-tubes or additives can also be employed. To decrease the mass load and increase efficiency; the different combinations of elements are substituted like Nickel-Titanium, Copper-AluminiumNickel and Copper-Zinc-Aluminium to obtain lightweight materials (Murray et al., 2019). The different materials utilized for the construction of the WT blade regarding the techno-economic model include Root Hardware, Gelcoat, Adhesives, Resin Systems, Fiber-Glass Fabric, Materials of Core, Lightning Protection System, Leading-Edge Protection, etc. (Dathu and Hariharan, 2020).

\section{Improving Blade Design}

In quest of getting a design that is optimal for composite offshore WT blades both structural properties and practical limiting conditions are considered and to achieve the objective work is executed in 2 stages particularly aerodynamic design and structural design. The aerodynamic performance according to the methodology of Blade Element Momentum (or BEM) estimates load over the blade, predicts the damage and turbine function even in adverse conditions. Also, an approach is fabricated to spot failure of large structures in sensitive areas, which supported non-linear finite element analysis, thereby determining an optimal blade design (Tarfaoui et al., 2019).

\section{Improvements in Structural Design}

To obtain the ratio of high stiffness to mass along with greater strength various composite structures are being used for different types of equipment in WT more emphasis is being laid over the susceptibility and micro damage of composite materials. Damages like fiber breakage and matrix crack will eventually cause a fracture in the macroscopic structure. A strategic approach for multi-scale modeling is discussed to detect mechanisms of failure. Hence, for composite materials method via $3 \mathrm{D}$ reverse modeling can be practiced to efficiently estimate their mechanical properties, thereby predicting and preventing failure. This will increase the reliability and safety in WT and promote a more effective, perhaps comprehensive blade design (Ye et al., 2019).

\section{Structural Analysis}

Stress and deformation analysis and study of vibrational characteristics based on the Finite element method were performed at maximum rated power was done at WTB parts made up of three different materials Carbon Fiber Reinforced (CFRP).

Plastic, Kevlar and E-Glass Fiber. Obtaining VonMises stress and total deformation it is revealed that CFRP is the optimum material with the minimum level of stress. Vibration analysis suggests the material with higher structural stiffness and which avoids high frequencies and mode shape is Carbon Fiber Reinforced Plastic. Wind loads lead to deflections in the WTB, therefore to avoid failure materials must be strong light in weight (Zuheir et al., 2019). A point-cloud to polysurface model is developed for the reconstruction of the geometry of a turbine blade digitally which would make it easy for architects, engineers to design, analyze and decide for parts of the blade when decommissioned, i.e., to be recycled or reused. To optimize best-fitting airfoil curves, steps are followed to minimize distances between the points on the curve and point-cloud cross-section (Tasistro-Hart et al., 2019).

\section{Preventing and Investigating Buckling (WTB)}

Trailing edges of WTB are vulnerable to buckling, provided that unsupported panels are subjected to compressive or shear loads and assessing the consequences of discrete material failures. The study gives deeper insights over crucial factors during compressive loading, which regulates buckling effect and the load-carrying capacity of the trailing edge. It is achieved by employing diverse FE functions and features. It has been noticed for the section of trailing edges, that there is a serious impact over the loadcarrying capacity from the failure of surface contact with foam core materials. Thereafter, structural reinforcement which consists of various reinforcement materials is applied at portions of the trailing edge with the existence of surface contact. Hence, a significant increase is evident in the load-carrying capacity of the structure engineered. To add strength we can use reinforcements in the WTB (Chen et al., 2019d). We can explore the technique or mechanism behind the structural failures of a trailing edge section of WTB, with the help of nonlinear Finite Element (FE) model upon the analysis of trends of progressive failure and continuum damage, in subcomponent testing with the help of Digital Image Correlation (or DIC) record deformations due to buckling and strain estimation. Along with buckling, the contact of the surface with the sandwich panels is responsible for the failure. At the maximum level (peak) of load-carrying capacity failure 
of foam material starts whereas, composite and adhesive materials fail after the peak (Chen et al., 2019c).

\section{Aero-Vortex Method with Neural Networks}

With the help of lifting-line theory, a vortex particle method is described along with the structural dynamics of WTB. Neural networks and gradientbased optimizer are of great help to simulate and design a complex aero-elastic wind-turbine. The new design includes more pre-bend and sweep than the straight-blade design. An increase of mean thrust by $0.02 \%$ and power output by nearly $1 \%$ is observed when the design is inclusive of turbulent in-flow conditions. With the extensive usage of Neural Networks for simulation it becomes easy to determine further improvements that can be made in the overall structural design (Sessarego et al., 2020).

\section{Vacuum Assisted Resin Infusion}

WTB design based on Triplaris Americana's (Tree Seed) curvature and balancing of various operational loads like aerodynamic and inertial are estimated. The new design of structural composites has 6 distinct zones for the lamination of the carbon fiber in the investigation. Study of vacuum assisted resin of infusion over the composites shows the net result in 4 different strategies that are possible for injection. 3 of them are in support of the objective, as it completely fills the mold and limits the time taken for the polymerization of the resin. The changes done tend to negligibly impact on decreasing the performance of the unit (Herrera et al., 2019).

\section{Improvements in Aerodynamic Design}

\section{Dynamic Analysis}

Dynamic analysis has always been behind various crucial properties cum parameters such as aeroelastic analysis, stability and flutter, delamination, transient and fatigue analysis. Modern deep neural networks like nonlinear autoregressive networks are used for forecasting wind speed and fatigue analysis. Hence, involving highfidelity modeling for the composite materials of the blade, an integrated methodology is developed to establish time-varying loads over blade cross-sections to calculate dynamic wind loads, using multiple environmental parameters and deep learning for wind speed forecasting methods and fatigue analysis for a WT blade using the stress life approach with load ratio based on cohesive zone modeling (Kulkarni et al., 2019).

\section{Gravo-Aeroelastic Scaling (GAS)}

An approach for the structural design of WT by developing a bio-inspirational method via GravoAeroelastic Scaling (GAS) (including the composition of various loads like that of aerodynamic, centrifugal and gravitational) for additive manufacturing. Hence, a model is designed to improve GAS performance by promoting the bio-inspirational reinforcements and lightweight morphology in the carbon fibre. The model discussed can be utilized to make a sub-scale turbine which small to an extent of $1 \%$ of an extreme scale turbine from the application of GAS concept (Kaminski et al., 2020).

\section{Optimization of Aerodynamic Design}

Aerodynamic design and efficiency, electricity generation and to minimize thrust force are key domains to be optimized for improvement in the aerodynamic design. In consideration of various practical and environmental factors, assessed by RSM geometry is optimized. Reflecting significant enhancement in the performance of the blade as efficiency grows nearly $10 \%$ with almost no change in the thrust force. It provides an optimal strategy, which is essentially proved to be a successful approach to optimize and improve the current resources, thereby net increase in efficiency and power output (Lee and Shin, 2020).

\section{Cavity Shape Optimization Approach}

To resolve the problem of optimal parameters lying beyond the plan, which is an issue for many perfectly designed conventional aerofoils, proposed is a cavity shaped over the aerofoils. The downstream suction surface and shape are parameterized to obtain an optimum lift-to-drag ratio. Thus, the cavity well drapes the vortex for stall margin regulation, reducing the impact of flow fluctuations and substantially improving the lift-to- drag ratio. The experiment gives evidence of 31 and $57 \%$ increase in lift-to-drag ratio when the angle of attack is about 14 and 20 degrees respectively (Fatehi et al., 2019).

\section{Environmental Impact}

The impact of adverse weather conditions predominantly for offshore projects or acid rain causes deterioration of WT components. Droplet erosion analysis of the WT blades (for Glass Fiber Reinforced Epoxy material), predicts corrosion for saline and acidic rain conditions. Thereby, affecting the material of the blade, also, the prime reason behind delamination inbetween the fiber layers and micro stresses leading to adhesion losses in reinforcement fibers (Rasool et al., 2020). Molecular level analysis alongside chemical degradation helps in observation of the electrical and thermal lightning impact over different core materials. Lightning strikes severely damage the blade materials.

\section{Preventing Thermal Damage from Lightening}

Various core materials like (Basla) wood, PVC and PET are subjected to impulse voltages to the level 
lightning in order to determine the characteristics of material behavior around the breakdown point. Blade's core of balsa wood is proved more probable to the breakdown and resistive towards the thermal effect of lightning discharge in comparison with other materials. Out of the three PVC material is more susceptible to the damage in terms of the thermal effect of lightning. Hence; operational safety is maximized based upon withstanding performance of core material employed (Yan et al., 2019).

\section{Surface Erosion}

As blades face harsh environmental conditions like humidity, rainwater, UV rays, sand-laden winds, sand abrasion, thunder strikes, etc. Chronic exposure to different temperatures and there changes significantly affect the blade material also deterioration of the aerodynamic performance. It increases the roughness of the blade surface and hence a reduction in power output. Rainwater causes erosion and scouring and also impacts the rotating speed of the WTB. Surface erosion of WTB leads to a reduction in the power production and lifespan of blade materials. We can analyze rain erosion mechanisms and develop a protection technology like protective comprehensive coating prepared by a combination of resins possessing different properties can be developed by considering the effect of the rainwater acidity over organic coatings (Chen et al., 2019a). Important parameters responsible for the erosion of the trailing edges of WTB are identified and then the behavior of the protective coating and its erosion performance is simulated. The characteristics of the desired coating material are obtained and the design required can further be investigated to meet the environmental and project objectives of the site (Doagou-Rad and Mishnaevsky, 2020).

\section{Preventing Surface Erosion}

Blades are prone to damages in composite materials due to leading-edge erosion leading to reduction in gross energy generation, like in case of offshore wind projects. Coating of blade using sol-gel technique is proved convenient with imparting negligible effect over the weight of WTB. They prevent rain-erosion and with the use of appropriate materials the losses can be significantly reduced. To enhance resistance against erosion, carbon nanotubes and graphene nano-additives can also be used (Dashtkar et al., 2019). The characteristics of desired coating material are obtained and the design required can further be investigated to meet the environmental and project objectives of the site (Doagou-Rad and Mishnaevsky, 2020). The different coatings if tested with relative Acoustic Impedance, then they contribute in increasing life span and strength of coating layers (Domenech et al., 2020).

\section{End of Life Options}

WT are composed of about $80-85 \%$ metal, hence recycling the unit after it is decommissioned will add on the sustainable and profitable strategy towards retaining the quality (Psomopoulos et al., 2019). A comparison for options regarding end-of-life for WT blade materials (specifically Glass Fibre Reinforced Plastic and Carbon Fibre-reinforced plastic), with respect to environmental impact, energy consumption and the benefits of recycling and the trends progressing for development in blade technology. The options available, rather achievable rely over material of the blade; generally are composites of glass and carbon fibre (Liu et al., 2019). The blade and other WT components are now enforced towards greener practices of composite waste treatment instead of incinerating or landfill. These new methods are quite similar for wastes from aerospace or automotive industries. The recycling technology for composite materials involve thermal, mechanical and chemical processes which tend to meet environmental regulations, optimized level of quality and cost. Majority of the Mechanical treatment is relatively simple, needs more energy and involves size reduction. The thermal and chemical recycling involves of the breakdown of chemicals at molecular level by means of high temperature, pyrolysis, de-polymerization and fluid-bed combustion (Kalkanis et al., 2019).

Seeking attention towards environmental and more optimized conservation of the resources one can opt to recycle and reuse the WT blades made up of thermoset composite materials. It involves insights towards affordable, ecofriendly and efficient re-processing or recycling and manufacturing technologies to promote and commercialize laboratory scale methods. Also utilizing ecofriendly materials like natural fiber, thermosetting resin (modified) and recyclable thermoplastic resin or design blades based on the performance of material for better efficiency (Chen et al., 2019b). Economically viable, a composite fabrication system is used to recycle Wind Turbine Blades (WTB) which are generally made up of glass fiber composites. Here, feedstock for composites of thermoplastic are mechanically recycled WTB materials and they are mechanically milled and classified from screening. They are blended with the thermoplastic resin of HighDensity Polyethylene (HDPE). Coefficient of Linear Thermal Expansion, thickness swell and water sorption properties and static bending is calculated for all composites along with thermal and thermogravimetric analytics. The result obtained proves feasibility of mechanical recycling of WTB and it to be a better method for fabrication of high-performance second gen. composite. Fiber reinforced composites are thus obtained from mechanical recycling of WTB materials (Mamanpush et al., 2019). 


\section{Wind Energy Economics}

With surge in Wind Energy as evident from the Fig. 3, the demand or further progress will aim materials of higher strength and which are fatigue resistant. With regular development or improvement in designs of turbines, it becomes crucial to update recycling technologies. Market of wind energy has continuously increased consumption of FRP composites across the globe, remarking the swift and steady progress in the sector (Psomopoulos et al., 2019). Key factors of the economics involved for wind energy are capital costs (that account for design and construction) which cover grid connection, civil engineering aspects and the institutional and machinery expenditures required for the establishment of wind energy projects. Whereas, there are variable costs involved for operation and maintenance that consists of capacity and efficiency of electricity production and transmission. Though, the theoretical energy generation is reduced by a variety of factors, like array losses - which occur because of wind turbines shadowing each other in a very powerful plantlike corrosion of blades, electrical losses in transmission and turbine periodic maintenance or technical glitches or failure. Hence, the gross generation is typically estimated at $10-15 \%$ below the energy calculation supported the turbine power curves provided.

There prevail substantial differences regarding the estimated investments required for onshore and offshore projects as of practical challenges that are faced in offshore wind project installation. Like foundations are the significantly expensive cause of cost dependency on both the water depth and suited principle of construction and, will be even higher in deeper water or with less favorable soil conditions. Moreover, due to adverse climate conditions, the operational and also the maintenance costs are high for offshore and with a view to establishing the long-term cost tendency of wind energy, one would want to differentiate the price elements that depend upon the wind resource and on the technological advancement that is determined by the local energy resources and taxation policies for the region where the wind park is placed. The lifetime of an onshore wind park is mostly assumed to be 20 years and 25-30 years for an offshore power station. However, some studies are supported different timespans, or presume longer or shorter periods for individual components. The Dutch Research Institute ECN has a public-access model that works with a 15 year lifetime. There are other models that have a productive lifespan of 16 to 24 years in the case of onshore and up to 40 years for offshore projects. The cost elements have seen a serious reduction in amounts for per $\mathrm{kWh}$ calculation as the supply chain involvement, favorable long term trends and perspective have led to enormous technical advancements in diversified sections for electricity generation and transmission for the same. There has been observed a continuous surge in wind energy demand by the last two decades and the turbines today produce about 180 times more energy at half the cost per kWh (Blanco, 2009).

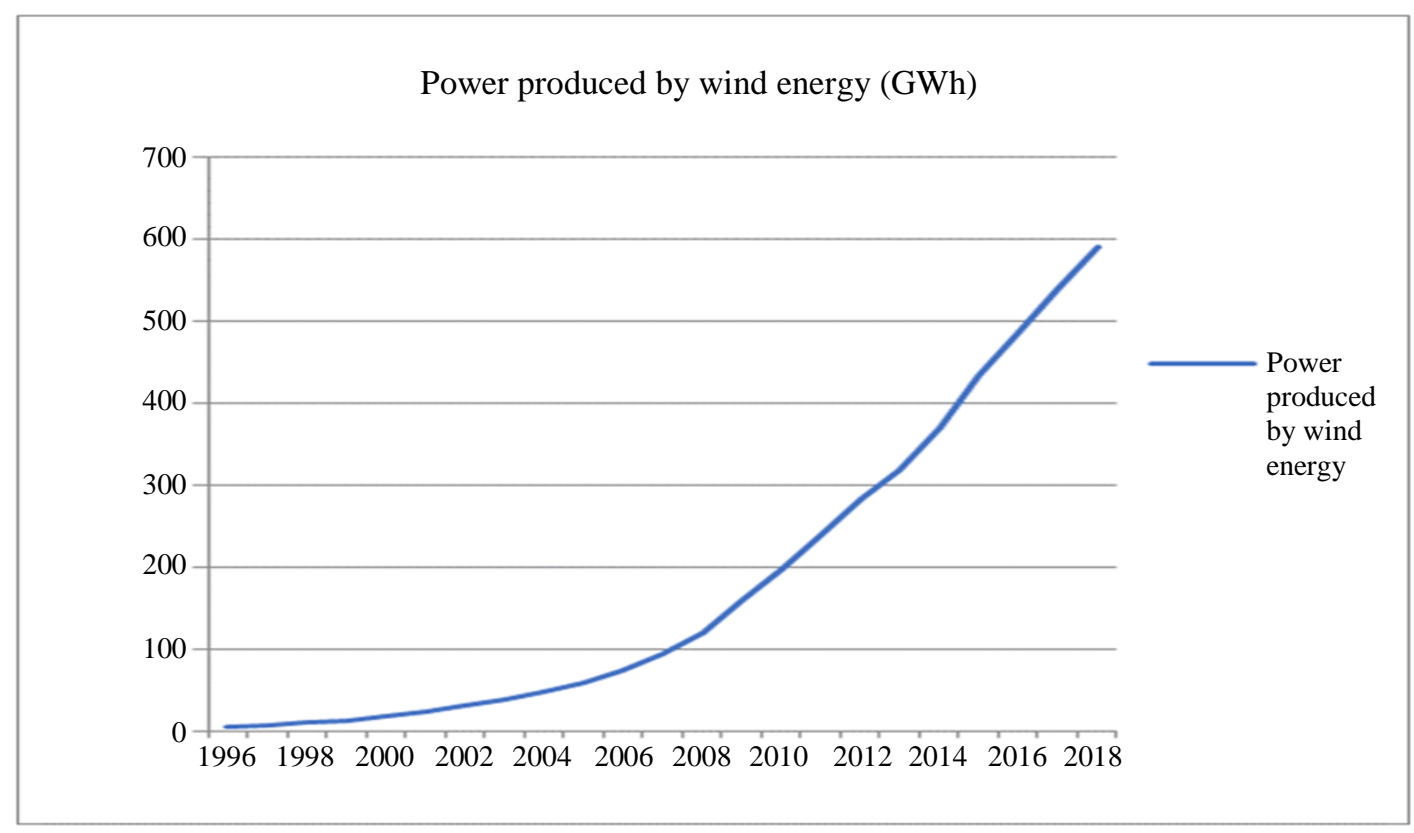

Fig. 3: Electricity output from wind energy 


\section{Conclusion}

To meet the exponentially surging demands in wind energy project it is crucial to use materials that enhance efficiency as well as justified in terms of costs. Carbon fibre is expensive yet efficient for the power production. To improve mechanical properties of the fibers, composites are reinforced with nanomaterial like CNT, etc. composites. Light-weight materials for WTB, reduces noise, increases the rotation speed but are expensive and generate heat a lot. Medium weight materials reduce noise, are long-lasting and their rotation velocity is more stabilized with respect to the wind speed. Less heat is produced due to smooth rotation. To decrease the mass load and increase efficiency; different combinations of elements are substituted like Nickel- Titanium, CopperAluminium-Nickel and Copper-Zinc- Aluminium to obtain lightweight materials. Blades of the WT accounts for the $15-20 \%$ of the unit cost. By improving the blade designs by using fine element, analysis, vibrational, fatigue and other analysis, the mechanical properties like structural and aerodynamics of the blade can be significantly upgraded. It has been noticed for the section of trailing edges, that there is a serious impact over the loadcarrying capacity from the failure of surface contact with foam core materials. Therefore with the help of appropriate buckling can be prevented. Using 1\% GAS, cavity shape optimization approach to improve lift to drag ratio, aero-vortex method for vacuum infusion of resins and measure as WTB are exposed to harsh environmental conditions the design of the WTB can be improved with net increase in power output. We can estimate the service life of the blades using computational simulation and forecast for the rotor speed can be obtained. Once the blade's lifespan is over it is important to dispose of them properly. To recycle the blade material which is generally carbon or glass fibre they are blended with thermoplastic resins. WT today produce 180 times more energy at half the costs thereby saving tons of $\mathrm{CO}_{2}$ every year.

\section{Acknowledgement}

The authors would like to thank National Institute of Technology, Tiruchirappalli for providing the necessary platform and support for carrying out this research.

\section{Author's Contributions}

Ojshwi Prakash: Preparing the manuscript.

Godwin Glivin: Designing the research plan.

N. Kalaiselvan: Guiding in research.

V. Mariappan: Overall supervision and checking.

\section{Ethics}

This article is original and contains unpublished works. The corresponding author confirms that all of the other authors have read and approved the manuscript with no ethical issues involved.

\section{References}

Blanco, M. I. (2009). The economics of wind energy. Renewable and sustainable energy reviews, 13(6-7), 1372-1382.

Chen, J., Wang, J., \& Ni, A. (2019a). A review on rain erosion protection of wind turbine blades. Journal of Coatings Technology and Research, 16(1), 15-24.

Chen, J., Wang, J., \& Ni, A. (2019b). Recycling and reuse of composite materials for wind turbine blades: An overview. Journal of Reinforced Plastics and Composites, 38(12), 567-577.

Chen, X., Berring, P., Madsen, S. H., Branner, K., \& Semenov, S. (2019c). Understanding progressive failure mechanisms of a wind turbine blade trailing edge section through subcomponent tests and non-linear FE analysis. Composite Structures, 214, 422-438.

Chen, X., Haselbach, P. U., Branner, K., \& Madsen, S. H. (2019d). Effects of different material failures and surface contact on structural response of trailing edge sections in composite wind turbine blades. Composite Structures, 226, 111306.

Dashtkar, A., Hadavinia, H., Sahinkaya, M. N., Williams, N. A., Vahid, S., Ismail, F., \& Turner, M. (2019). Rain erosion-resistant coatings for wind turbine blades: A review. Polymers and Polymer Composites, 27(8), 443-475.

Dathu, K. P. M. Y. V., \& Hariharan, R. (2020). Design of wind turbine blade material for higher efficiency. Materials Today: Proceedings.

Doagou-Rad, S., \& Mishnaevsky, L. (2020). Rain erosion of wind turbine blades: Computational analysis of parameters controlling the surface degradation. Meccanica, 55(4), 725-743.

Domenech, L., Renau, J., Šakalytè, A., \& Sánchez, F. (2020). Top Coating Anti-Erosion Performance Analysis in Wind Turbine Blades Depending on Relative Acoustic Impedance. Part 1: Modelling Approach. Coatings, 10(7), 685.

Ennis, B. L., Kelley, C. L., Naughton, B. T., Norris, B., Das, S., Lee, D., \& Miller, D. (2019). Optimized Carbon Fiber Composites in Wind Turbine Blade Design (No. SAND2019-14173). Sandia National Lab.(SNL-NM), Albuquerque, NM (United States).

Fatehi, M., Nili-Ahmadabadi, M., Nematollahi, O., Minaiean, A., \& Kim, K. C. (2019). Aerodynamic performance improvement of wind turbine blade by cavity shape optimization. Renewable Energy, 132, 773-785. 
Herbert, G. J., Iniyan, S., Sreevalsan, E., \& Rajapandian, S. (2007). A review of wind energy technologies. Renewable and sustainable energy Reviews, 11(6), 1117-1145.

Herrera, C., Correa, M., Villada, V., Vanegas, J. D., García, J. G., Nieto-Londoño, C., \& Sierra-Pérez, J. (2019). Structural design and manufacturing process of a low scale bio-inspired wind turbine blades. Composite Structures, 208, 1-12.

Kalkanis, K., Psomopoulos, C. S., Kaminaris, S., Ioannidis, G., \& Pachos, P. (2019). Wind turbine blade composite materials-End of life treatment methods. Energy Procedia, 157, 1136-1143.

Kaminski, M., Loth, E., Griffith, D. T., \& Qin, C. C. (2020). Ground testing of a $1 \%$ gravo-aeroelastically scaled additively-manufactured wind turbine blade with bio-inspired structural design. Renewable Energy, 148, 639-650.

Kulkarni, P. A., Dhoble, A. S., \& Padole, P. M. (2019). Deep neural network-based wind speed forecasting and fatigue analysis of a large composite wind turbine blade. Proceedings of the Institution of Mechanical Engineers, Part $\mathrm{C}$ : Journal of Mechanical Engineering Science, 233(8), 2794-2812.

Lee, S. L., \& Shin, S. (2020). Wind Turbine Blade Optimal Design Considering Multi-Parameters and Response Surface Method. Energies, 13(7), 1639.

Liu, P., Meng, F., \& Barlow, C. Y. (2019). Wind turbine blade end-of-life options: An eco-audit comparison. Journal of Cleaner Production, 212, 1268-1281.

Mamanpush, S. H., Li, H., Englund, K., \& Tabatabaei, A. T. (2019). Extruded fiber-reinforced composites manufactured from recycled wind turbine blade material. Waste and biomass valorization, 1-10.

Murray, R. E., Jenne, S., Snowberg, D., Berry, D., \& Cousins, D. (2019). Techno-economic analysis of a megawatt-scale thermoplastic resin wind turbine blade. Renewable Energy, 131, 111-119.

Pourrajabian, A., Dehghan, M., Javed, A., \& Wood, D. (2019). Choosing an appropriate timber for a small wind turbine blade: A comparative study. Renewable and Sustainable Energy Reviews, 100, 1-8.

Pradeep, A. V., Prasad, S. S., Suryam, L. V., \& Kumari, P. P. (2019). A comprehensive review on contemporary materials used for blades of wind turbine. Materials Today: Proceedings, 19, 556-559.

Psomopoulos, C. S., Kalkanis, K., Kaminaris, S., Ioannidis, G. C., \& Pachos, P. (2019). A Review of the Potential for the Recovery of Wind Turbine Blade Waste Materials. Recycling, 4(1), 7.
Rasool, G., Middleton, A. C., \& Stack, M. M. (2020). Mapping Raindrop Erosion of GFRP Composite Wind Turbine Blade Materials: Perspectives on Degradation Effects in Offshore and Acid Rain Environmental Conditions. Journal of Tribology, 142(6).

Schubel, P. J., \& Crossley, R. J. (2012). Wind turbine blade design. Energies, 5(9), 3425-3449.

Sessarego, M., Feng, J., Ramos-García, N., \& Horcas, S. G. (2020). Design optimization of a curved wind turbine blade using neural networks and an aeroelastic vortex method under turbulent inflow. Renewable Energy, 146, 1524-1535.

Tarfaoui, M., Shah, O. R., \& Nachtane, M. (2019). Design and optimization of composite offshore wind turbine blades. Journal of Energy Resources Technology, 141(5).

Tasistro-Hart, B., Al-Haddad, T., Bank, L. C., \& Gentry, R. (2019). Reconstruction of Wind Turbine Blade Geometry and Internal Structure from Point Cloud Data. In Computing in Civil Engineering 2019: Data, Sensing and Analytics (pp. 130-137). Reston, VA: American Society of Civil Engineers.

Vermeer, L. J., Sørensen, J. N., \& Crespo, A. (2003). Wind turbine wake aerodynamics. Progress in aerospace sciences, 39(6-7), 467-510.

Wu, Y. T., Liao, T. L., Chen, C. K., Lin, C. Y., \& Chen, P. W. (2019). Power output efficiency in large wind farms with different hub heights and configurations. Renewable Energy, 132, 941-949.

Yan, J., Wang, G., Ma, Y., Guo, Z., Ren, H., Zhang, L., ... \& Yan, J. D. (2019). Electrical and thermal performance of different core materials applied in wind turbine blades under lightning strikes. Wind Energy, 22(11), 1603-1621.

Ye, J., Chu, C., Cai, H., Hou, X., Shi, B., Tian, S., ... \& Ye, J. (2019). A multi-scale model for studying failure mechanisms of composite wind turbine blades. Composite Structures, 212, 220-229.

Zuheir, S., Abdullah, O. I., \& Al-Maliki, M. (2019). Stress and vibration analyses of the wind turbine blade (A NREL 5MW). Journal of mechanical engineering research and developments, 42(4), 14-19. 